\title{
Making Space for the Psychology of Creativity in Dance Science
}

Lucie Clements, Trinity Laban Conservatoire of Music \& Dance, London, UK

Rebecca Weber, Coventry University, London, UK

\section{ABSTRACT}

Creativity is commonly recognized as a complex phenomenon; one which entails a range of debates around definition, process and product, domain specificity, crossdiscipline generalisability, and appropriate testing measures. The psychology of creativity appears to find a fitting home in dance science, a field concerned with understanding and enhancing dancers' health and performance. Yet dance psychology has been predominated by research which focuses on the mental processes underpinning optimal skill execution and technical performance. This paper outlines an argument for a greater focus on the creative demands of dance within dance science, highlighting some the challenges of, and barriers to, research in the psychology of creativity in dance, before making a number of recommendations to encourage the growth of this important research area.

\section{KEYWORDS}

Cognitive Psychology, Collaboration, Creativity, Dance Science, Imagination, Interdisciplinary, Psychology 


\section{INTRODUCTION}

The study of the psychology of creativity in dance appears to find a fitting home in dance science, a field invested in the application of a broad range of sciences to understand and enhance the art of dance. Dance science is concerned with encouraging safe practice, enhancing well-being, and optimizing performance through "scientific knowledge and evidence-based practice" (National Institute of Dance Medicine and Science, 2015). As such, psychology forms one subset of dance science (International Association of Dance Medicine and Science, 2017). The study of creativity, as a research discipline unto itself, is situated in the field of cognitive psychology; therefore, with its melding of psychological sciences and dance, research on creativity in dance should find a suitable home in the dance science field. In recognition of, and in response to, a concern that creativity as an area has been neglected in dance science, this article presents a status quo review (Noguchi, 2006) of creativity research. We are informed, in equal parts, by peer-reviewed literature from the science and dance fields to make an argument for the necessity of creativity at a topic in dance science. First, we outline our ideas regarding the field's existing emphasis on technique over creativity enhancement in dance. Next, we discuss the perceived issues and challenges of studying creativity in dance science, with a particular focus on definition, measurement and the non-verbal, embodied and gestalt nature of dance. We then propose some recommendations for future research, drawing on our experience as both dancers and scientists.

\section{BACKGROUND}

The current dance ecology sees the relationship between choreographer and dancer as blurred, with the choreographer no longer deemed the sole creative (Farrer, 2014). It is common within contemporary dance that dancers contribute to the development of movement material (Butterworth, 2004; Rowe \& Smith, 2011), and in many contemporary practices, the hierarchical norm and dichotomous separation of dancer and choreographer is dissolved. As more dance professionals seek a portfolio career, dance education programmes emphasize the dual aims of developing technique and creativity (Bennett, 2009). Indeed, creativity is considered an important facet of talent in dance (Redding, Nordin-Bates \& Walker, 2011). Thus, we argue that creativity is of relevance for all dance artists, and is a pertinent topic for dance science.

Despite its importance and the fact that dance is recognized as a creative activity, there is a lack of research on the psychology of creativity in the dancer's training and career. The relevance of inter- and intrapersonal, environmental, and situational psychology in achieving optimal creativity in dance is unknown. Unsurprisingly then, dance science is yet to recognize the dancer as a creative artist, and further still, to understand the relevance of creativity within the health and well-being of dancers. It is clear that there is a place for creativity research within dance science. Potential areas of investigation include: 
14 more pages are available in the full version of this document, which may be purchased using the "Add to Cart" button on the publisher's webpage: www.igiglobal.com/article/making-space-for-the-psychology-ofcreativity-in-dance-science/211119

\title{
Related Content
}

\begin{abstract}
Accurate Infrared Tracking System for Immersive Virtual Environments Filipe Gaspar, Rafael Bastos and Miguel Sales (2012). Innovative Design and Creation of Visual Interfaces: Advancements and Trends (pp. 318-343). www.irma-international.org/chapter/accurate-infrared-tracking-system-immersive/64061
\end{abstract}

Found Objects, Bought Selves

Lynne Heller (2015). New Opportunities for Artistic Practice in Virtual Worlds (pp. 140-161).

www.irma-international.org/chapter/found-objects-bought-selves/132421

Perspectives on Hyper-Realities: Simulacra and Shadows of Silent Gazes Albert Samuel Nii Sackey, Edwin Kwesi Bodjawah, Samuel Nortey and George Ampratwum (2021). International Journal of Art, Culture and Design Technologies (pp. 41-56).

www.irma-international.org/article/perspectives-on-hyper-realities/297019

Data-Driven Maps of Art History

Doron Goldfarb and Dieter Merkl (2019). International Journal of Art, Culture, Design, and Technology (pp. 1-15).

www.irma-international.org/article/data-driven-maps-of-art-history/233614

Designing Dynamic Logotypes to Represent Data Jéssica Parente, Tiago Martins, João Bicker and Penousal Machado (2019). International Journal of Art, Culture, Design, and Technology (pp. 16-30).

www.irma-international.org/article/designing-dynamic-logotypes-to-represent-data/233615 